\title{
Plasma Surface Modification of Polymer Substrate for Cell Adhesion Control
}

\author{
Yasushi Sasai, Shin-ichi Kondo, Yukinori Yamauchi ${ }^{\dagger}$ and Masayuki Kuzuya ${ }^{\dagger}$ \\ Laboratory of Pharmaceutical Physical Chemistry, Department of Drug Delivery Technology and Science, \\ Gifu Pharmaceutical University, 1-25-4 Daigaku-Nishi, Gifu 501-1196, Japan \\ ${ }^{\dagger}$ Department of Pharmaceutical Physical Chemistry, College of Pharmaceutical Sciences, \\ Matsuyama University, 4-2 Bunkyo-cho, Matsuyama, Ehime 790-8578 Japan \\ sasai@gifu-pu.ac.jp
}

Keywords: plasma techniques, vinylmethylether maleic anhydride copolymer, polystyrene, cell adhesion peptide

\section{Introduction}

Since the surface treatment by low temperature plasmas can vary the physicochemical, structural and functional properties on polymer material without affecting the bulk characteristics, it has been widely used to control of the biocompatibility of polymeric materials. [1-3] For example, the cell adhesion onto polymer substrate is enhanced by hydrophilization on the surface using plasma surface treatment. $[4,5]$ On the other hand, the hydrophobic recovery is commonly observed on the plasma irradiated polymer surface, which is a matter of great concern in the practical use. $[6,7]$

We have developed the method to introduce a durable surface hydrophilicity and minimize its decay with time on several hydrophobic polymers using plasma techniques. [8-10] This method involves a sorption of vinylmethylether-maleic anhydride copolymer (VEMA) into the surface layer of polymer substrate and the immobilization of VEMA by plasma-induced crosslink reaction, followed by hydrolysis of VEMA to generate hydrophilic carboxyl groups (VEMAC) on the surface. The conceptual procedure is sequentially illustrated in Fig. 1. The VEMAC-immobilized surface can also be used as a substrate for the covalent immobilization of the bioactive molecules through reactions with carboxyl group.

In our previous paper, we reported the preparation of VEMAC-immobilized polystyrene

\begin{tabular}{lll}
\hline Received & April & 16,2010 \\
Accepted & May & 21,2010
\end{tabular}

(PS/VEMAC) dish by essentially the same method showed in Fig. 1. [11] and the immobilization of cell adhesive peptide, Glycine-Arginine-GlycineAspartic acid-Serine (GRGDS) known as a fibronectin active fragment, on the surface [12]. The surface thus prepared promoted the adhesion and proliferation of mouse embryonic fibroblast, NIH3T3, in the culture with medium containing $10 \%$ calf serum.

When the substrate is exposed to a suspension of cells in culture medium containing serum, the serum proteins rapidly adsorb onto the surface, and

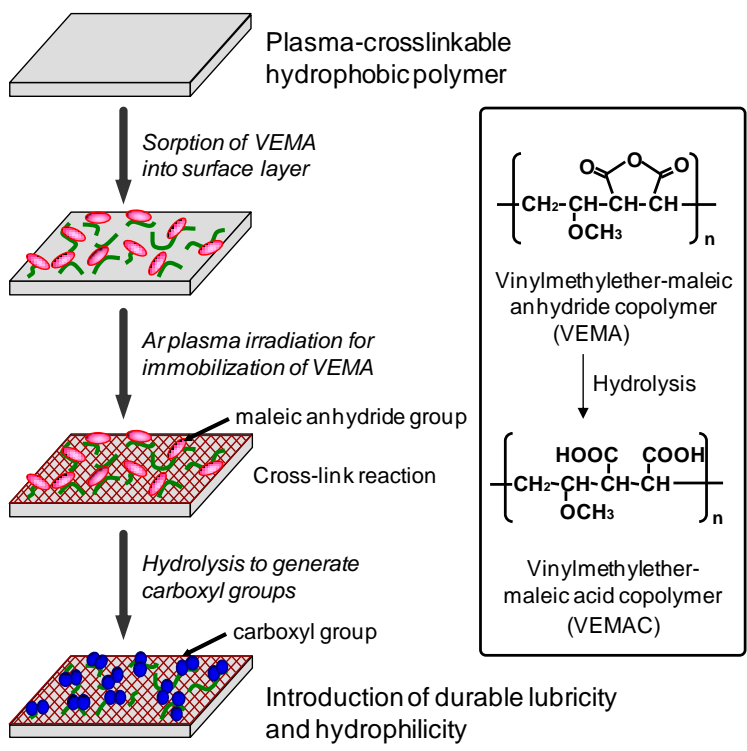

Fig. 1 Schematic illustration of fabrication of durable hydrophilicity on a polymer surface. 
then cells subsequently approach and settle on the surface. Thus, the serum proteins play important roles in the cell adhesion onto the substrate and affect the cell behavior and function. On the other hand, the use of animal-origin serum in the cell culture contains some potential risks, such as the batch-to-batch variability in composition and the contamination by adventitious agent. In addition, since the serum is a complex mixture of many and various constituents, the effect of serum components on cultured cells often remains unclear.

The aim of this study is to fabricate a cell culture substrate with cell adhesive property under serumfree conditions. We applied the GRGDSimmobilized PS/VEMAC dish in serum-free cell culture and examined the effect of the immobilized GRGDS peptide on the cell adhesion.

\section{Experimental}

\subsection{Preparation of PS/VEMAC dish}

A commercial polystyrene (PS) dish $(35 \mathrm{~mm}$ in diameter) was cleaned by ultrasonication in methanol and dried in vacuo at room temperature. $1 \%(\mathrm{w} / \mathrm{v})$ VEMA solution of dimethyl sulfoxide (DMSO) was added in the non-treated PS dish and it was incubated for $1 \mathrm{~h}$ at $30{ }^{\circ} \mathrm{C}$ to penetrate VEMA into PS surface layer. Then, the PS dish was washed with DMSO to remove the excess VEMA on the surface. After drying at $50{ }^{\circ} \mathrm{C}$ in vacuo for more than 10 hours, the PS dish thus treated was submitted to Ar plasma irradiation to immobilize VEMA onto the surface layer. The plasma state was generated by the use of radio-frequency discharge of inductive coupling with five loop antenna at $13.56 \mathrm{MHz}$ with the prescribed power. Flow volume $(50 \mathrm{ml} / \mathrm{min})$ and pressure $(66.6 \mathrm{~Pa}(0.5$ Torr $))$ of argon gas were controlled by flow meter and evacuating speed. The sample was placed in the reaction chamber (230 $\mathrm{mm}$ long, $45 \mathrm{~mm}$ in diameter) to ensure homogeneous exposure to plasma gas. After plasma irradiation, the hydrolysis of the maleic anhydride linkage in VEMA was conducted by immersing the PS dish immobilizing VEMA (PS/VEMA) in $0.1 \mathrm{M} \mathrm{NaOH}$ solution for 10 minutes. The hydrolyzed PS/VEMA dish was soaked in $1 \mathrm{M} \mathrm{HCl}$ for 10 minutes at room temperature and washed with distilled water to obtain PS/VEMAC dish. The procedure was essentially the same as that reported earlier. [11,12] We have previously reported that the surface density of carboxyl group on PS/VEMAC is affected by the plasma conditions for the immobilization of VEMA on PS substrate. [11] In this study, Ar plasma irradiation was conducted in the condition with a power of $30 \mathrm{~W}$ and a duration of 30s under our experimental setup. The density of carboxyl groups on the PS/VEMAC surface thus prepared was ca. $0.4 \mathrm{nmol} / \mathrm{cm}^{2}$.

\subsection{Immobilization of GRGDS peptide on PS/ VEMAC dish}

The covalent immobilization of GRGDS peptide on PS/VEMAC dish was carried out as follows: 50 $\mathrm{mM}$ phosphate buffer solution (PBS, pH5.8) containing $0.2 \mathrm{mM}$ 1-ethyl-3-(3-dimethyl-aminopropyl) carbodiimide hydrochloride (EDC) and 0.1 $\mathrm{mM}$ N-hydroxysulfosuccinimide (sulfo-NHS) was poured onto PS/VEMAC dish and incubate at 25 ${ }^{\circ} \mathrm{C}$ for $3 \mathrm{~h}$ with gently agitation to activate carboxyl group on PS/VEMAC dish. After washing with distilled water, $1 \mathrm{mg} / \mathrm{mL}$ GRGDS solution in PBS was poured onto the dish and incubated at $25{ }^{\circ} \mathrm{C}$ for $24 \mathrm{~h}$ to immobilize GRGDS peptide on the PS/VEMAC dish. The dish thus prepared was washed with Milli-Q water and then dried at room temperature. As the control experiment, GlycineArginine-Glycine-Glutamic acid-Serine (GRGES) peptide immobilized PS/VEMAC dish was prepared by the same method as described above. The peptide-immobilized dishes were sterilized by UV irradiation before use.

\subsection{Cell culture}

NIH3T3, mouse embryonic fibroblast cell line, was used as a model anchorage-dependent cell for cell adhesion test. NIH3T3 was routinely cultured in Dulbecco's modified Eagle medium (DMEM) supplemented with $10 \%$ calf serum, 100 units $/ \mathrm{mL}$ penicillin and $100 \mu \mathrm{g} / \mathrm{mL}$ streptomycin at $37{ }^{\circ} \mathrm{C}$ under a humidified atmosphere of $5 \% \mathrm{CO}_{2}$. After trypsin treatment, the cell suspension of the prescribed concentration was prepared with serumfree DMEM and was seeded into each dish. At a given time, the behavior of cell adhesion on the substrate was observed with phase contrast microscope and the number of cells adhered on each dish was determined by the Cell Proliferation Reagent WST-1 assay (Dojindo Laboratories).

\section{Results and Discussion}

Cell adhesion behavior on GRGDS-immobilized surface was compared with those on a commercial Type I collagen-coated PS and GRGESimmobilized surface under serum-free culture 
A. $2 \mathrm{~h}$

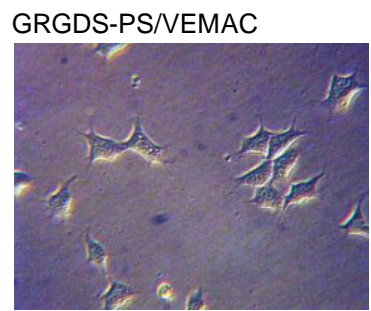

GRGDS-PS/VEMAC

B. $48 \mathrm{~h}$

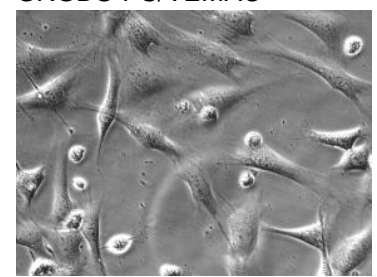

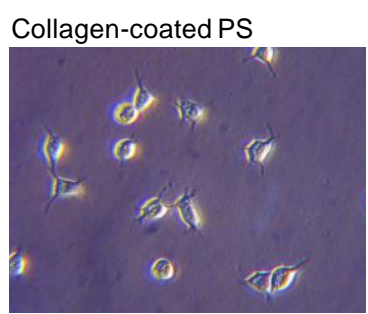

Collagen-coated PS

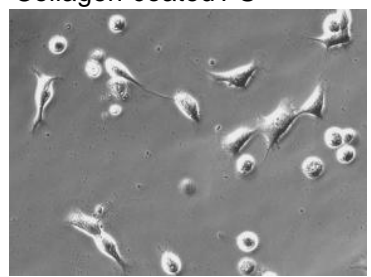

GRGES-PS/VEMAC

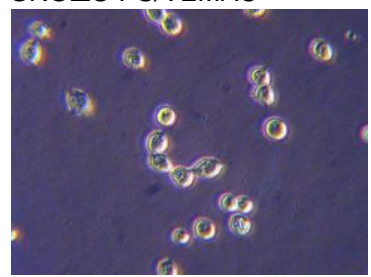

GRGES-PS/VEMAC

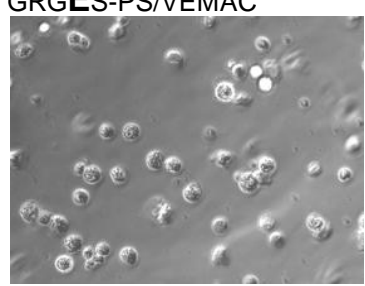

Fig. 2 Photomicrographs of NIH3T3 cells cultured for $2 \mathrm{~h}(\mathrm{~A})$ and $48 \mathrm{~h}$ (B) on various substrates. Number of seeded cells: 1.0 x $10^{5}$ cells.

conditions. Collagen is a major component of extracellular matrix (ECM) and serves many functions such as providing support and anchorage for cells and regulating intercellular communication. Therefore, the collagen-coating is used to promote cell adhesion and spreading on the culture substrate.

Figure 2 shows photomicrographs of NIH3T3 cells on each substrate after 2 hours (Fig. 2A) and 2 days (Fig. 2B) of culture. In this experiment, as the cells were cultured in serum-free medium, the initial adhesion must be occurred by the direct interaction between cell surface and substrate surface. As shown in Fig. 2A, the cells on the GRGDS-immobilized surface adhered and begun to spread on the surface at $2 \mathrm{~h}$ after seeding. On the other hand, few or no cells adhered onto collagen-coated and GRGES-immobilized surface. The promotional effect of the immobilized GRGDS-peptide on adhesion and spreading of NIH3T3 cells was more apparent at 2 days of culture as shown in Fig. 2B. The RGD sequence has been identified as a minimal essential cell adhesion peptide sequence in many ECM proteins and is recognized by cell surface receptor, integrin. $[13,14]$ These results shown in Fig. 2, therefore, indicate that the initial cell adhesion onto GRGDS-immobilized surface results from the specific interaction between RGD sequence in immobilized peptide and integrins.

The cell proliferation on the each substrate under serum-free condition was measured at 2 days. Figure 3 shows the number of cells adhered on the substrate after 2 days of culture in serum-free medium, together with that in medium with $10 \%$

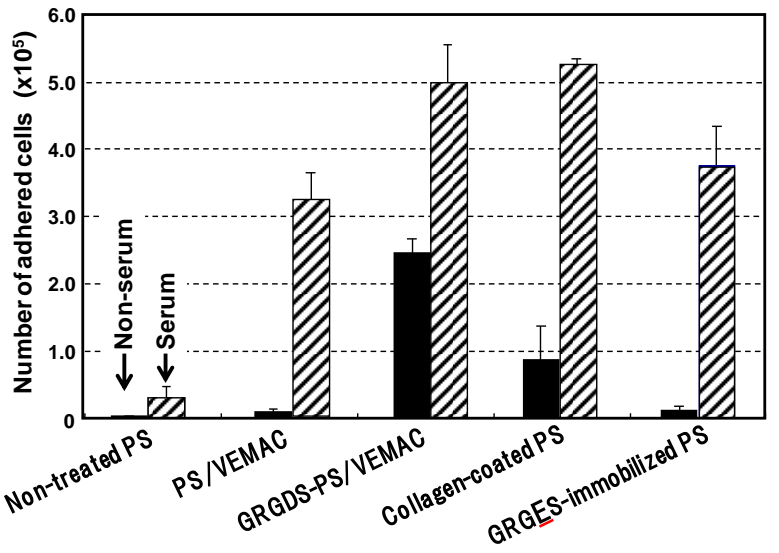

Fig. 3 Number of adhered NIH3T3 cells on various substrate at $48 \mathrm{~h}$ of cell culture with serum-free medium and $10 \%$ calf serum-containing medium.

Number of seeded cells: 1.0 x $10^{5}$ cells.

calf serum for the comparison purpose. Since the initial seeding density of cells was $1.0 \times 10^{5}$ cells/dish, the cell proliferation was observed only on the GRGDS-immobilized surface under serumfree conditions. These results suggest that the GRGDS-immobilized PS/ VEMAC surface works as artificial ECM and interacts with cell surface integrins to stimulate biological processes of cell such as growth and proliferation. On the other hand, the significant proliferation of cells cultured in serum-containing medium was observed on all substrates except for non-treated PS. This result indicates that the cell adhesion and proliferation on culture substrate in presence of serum are predominantly caused and promoted by serum. 


\section{Conclusion}

In this study, the cell adhesion properties on GRGDS-immobilized PS/VEMAC were examined under serum-free conditions. The result indicates that the promotional effects of immobilized GRGDS on cell adhesion and spreading are caused by a specific interaction of RGD peptide sequence and cell surface integrin receptor. The present study suggests that cell behaviors such as adhesion, spreading and proliferation can be control on the GRGDS-immobilized PS/VEMAC under serumfree conditions.

\section{Acknowledgment}

This work was financially supported in part by a Grant-in-Aid for Scientific Research from the Ministry of Education, Culture, Sports, Science and Technology of Japan (Grant No. 21790038), which is gratefully acknowledged.

\section{References}

1. R. Latkany, A. Tsuk, M. S. Sheu, I. H. Loh, V. Trinkaus-Randall, J. Biomed. Mater. Res., 36 (1997) 29.

2. T. G. van Looten, H. T. Spijeker, H. J. Busscher,
Biomaterials, 25 (2004) 1735.

3. N. P Rhodes, D. J. Willson, R. L. Williams, Biomaterials, 28 (2007) 4561.

4. J. Lee, J. Park, H. Lee, Biomaterials, 12 (1991) 443.

5. Y. Tamada, Y. Ikada, Polymer, 34 (1993) 2208.

6. Y. Lee, S. Han, J. Lee, J. Yoon, H. Lim, K. Kim, J. Vac. Sci. Technol., A 16 (1998) 1710.

7. C. C. Dupont-Gillain, Y. Adriaensen, S. Derclaye, P. G. Rouxhet, Langmuir, 16 (2000) 8194.

8. M. Kuzuya, T. Yamashiro, S. Kondo, M. Tsuiki, Plasmas and Polymers, 2 (1997) 133.

9. M. Kuzuya, T. Sawa, T. Yamashiro, S. Kondo, O. Takai, J. Photopolym. Sci. Technol., 14 (2001) 87.

10. M. Kuzuya, T. Sawa, M. Mouri, S. Kondo, O. Takai, Sur. Coat. Technol., 169-170 (2003) 587.

11. Y. Sasai, N. Matsuzaki, S. Kondo, M. Kuzuya, Sur. Coat. Technol., 202 (2008) 5724.

12. Y. Sasai, S. Kondo, Y. Yamasuchi, M. Kuzuya, J. Photopolym. Sci. Technol., 22 (2009) 503.

13. M. D. Pierschbacher, E. Ruoslahti, Nature, 309 (1984) 30.

14. M. Pfaff, In: J. A. Eble (Ed.), Integrin-Ligand Interaction, Springer-Verlag, Heidelberg (1997). 